\title{
Recherche en réanimation : enquête auprès des jeunes réanimateurs
}

\author{
Research in the Intensive Care Unit: a Survey of Young Intensive Care Physicians
}

\author{
Commission jeunes de la SRLF • P.-M. Bertrand - A. Beurton - L. Contentin - P.-É. Danin • E. Dubois • \\ J. Boisrame-Helms $\cdot$ É. Gélisse $\cdot$ Y. Karaca $\cdot$ P. Mora $\cdot$ N. de Prost $\cdot$ D. Roux $\cdot$ A. Soummer $\cdot$ D. Vovodar
}

(C) SRLF et Lavoisier SAS 2015

Pour un jeune réanimateur, la « Recherche » peut se présenter sous la forme de recherche clinique, au lit du malade, mais également sous la forme de recherche fondamentale pouvant s'intégrer dans le cadre d'un master 2 et d'une thèse d'université. La recherche prend aujourd'hui une importance croissante dans la formation et l'activité des réanimateurs. Ils sont ainsi amenés à participer activement à de nombreux protocoles de recherche clinique. Par ailleurs, la validation d'un master 2 pourrait devenir un prérequis indispensable pour l'accès à un poste de chef de clinique assistant. Partant de ce constat, la commission jeunes de la SRLF a interrogé les jeunes réanimateurs sur leur vision de la recherche.

En juin 2014, un questionnaire de 37 items a été envoyé par courriel à l'ensemble des membres jeunes de la SRLF, soit 1088 personnes de moins de 35 ans (un tiers des effectifs de la SRLF). Le taux de réponse a été de $24 \%$. Les membres jeunes répondeurs étaient comparables aux non-répondeurs en termes de sex-ratio $(\mathrm{F} / \mathrm{H}=0,7)$, d'origine géographique ( $89 \%$ de résidents en France répartis dans l'ensemble des interrégions), de statut (65\% en post-internat : $35 \%$ d'internes) et de spécialité initiale (anesthésie-réanimation : $56 \%$; spécialités médicales donnant accès au DESC de réanimation : $29 \%$; pédiatrie : $7 \%$; et de médecine d'urgence/ médecine générale : $6 \%$ ).

Trois éléments se sont dégagés dans les réponses obtenues :

- le premier constat est l'implication forte des jeunes réanimateurs dans la recherche clinique, puisque $86 \%$ déclaraient avoir déjà participé à un protocole de recherche biomédicale, soit de manière informelle $(33 \%)$, soit en tant que co-investigateurs $(46 \%)$ ou associés scientifiques $(7 \%)$.

Commission jeunes de la SRLF $(\bowtie) \cdot$ P.-M. Bertrand ·

A. Beurton $\cdot$ L. Contentin · P.-É. Danin · E. Dubois ·

J. Boisrame-Helms · É. Gélisse · Y. Karaca · P. Mora ·

N. de Prost $\cdot$ D. Roux $\cdot$ A. Soummer $\cdot$ D. Vovodar

Service de réanimation médicochirurgicale,

hôpital Louis-Mourier, 178, rue des renouillers,

F-92700 Colombes, France

e-mail : damien.roux@gmail.com
Si $44 \%$ des répondeurs caractérisaient la recherche comme indispensable en plus d'une activité clinique, seul $1 \%$ la jugeait inutile, le reste des répondeurs la jugeant facultative. Cette implication dans la recherche se traduisait pour $60 \%$ des répondeurs par une association lors de la publication des résultats comme coauteurs ou collaborateurs ;

- le deuxième constat est qu'un peu plus d'un tiers des répondeurs effectuaient ou avaient effectué un diplôme universitaire (36\% pour le master $2,16 \%$ pour la thèse d'université) et que la possibilité de réaliser un de ces deux diplômes était en majorité connue des répondeurs ( $73 \%$ pour le master $2,60 \%$ pour la thèse d'université). Les deux principales motivations chez les répondeurs ayant suivi un de ces deux diplômes étaient l'appétence intellectuelle $(50 \%)$ et la nécessité d'obtenir un de ces deux diplômes pour accéder à des postes universitaires (35\% pour la réalisation d'un master 2 et $48 \%$ pour la réalisation d'une thèse d'université). Ces diplômes étaient en très grande majorité réalisés dans l'interrégion d'origine, alors qu'une minorité les avait effectués à l'étranger ( $4 \%$ des masters 2 et $15 \%$ des thèses d'université) ;

- enfin, le troisième constat était la difficulté pour les jeunes de mener à bien un projet de master 2 ou de thèse d'université. Parmi les répondeurs, $61 \%$ avaient rencontré des difficultés, les principales étant le financement $(67 \%)$ et la difficulté de se dégager d'une activité clinique (20\%). Soixante et un pour cent des jeunes ayant réalisé ou réalisant un master 2 bénéficiaient d'un financement institutionnel, contre seulement $21 \%$ des répondeurs effectuant ou ayant effectué une thèse d'université. La difficulté dans le financement se traduisait par:

- la poursuite d'une activité clinique pour, respectivement, 36 et $70 \%$ des répondeurs ayant réalisé/réalisant un master 2 et/ou une thèse d'université sans pouvoir se consacrer exclusivement à une activité de recherche ;

- la faible part de recherche fondamentale ou translationnelle chez les répondeurs effectuant ou ayant effectué une thèse $(36 \%)$, qui nécessite le plus souvent une présence constante au laboratoire et l'interruption passagère de toute activité clinique. 
Cette enquête montre que les membres jeunes de la SRLF ont une vision globalement positive de la recherche. Il existe probablement un biais de sélection, puisque les jeunes réanimateurs non inscrits à la SRLF, comme les non-répondeurs à ce questionnaire, ont peut-être moins d'engagement dans la recherche que les répondeurs et les inscrits. Néanmoins, $55 \%$ des répondeurs jugent la recherche clinique facultative, alors qu'elle paraît indispensable à l'amélioration continue des soins. Le master 2 et la thèse d'université semblent soulever l'intérêt des jeunes réanimateurs, plus par appétence intellectuelle que pour l'accès à des fonctions universitaires. Le principal problème reste l'organisation pratique de ces activités de recherche, et notamment son financement, qui limite la possibilité d'une interruption temporaire de l'activité clinique pour se consacrer exclusivement à la recherche fondamentale.

Liens d'intérêts : La commission jeunes de la SRLF déclare ne pas avoir de lien d'intérêt. 